\title{
VISUALIZATION OF BROADBAND SOUND SOURCES
}

\author{
Dmitry Sukhanov ${ }^{\mathrm{a},}$ Nadezhda Erzakova \\ Radiophysics Faculty, Tomsk State University, 634050, Tomsk, Russia
}

\begin{abstract}
In this paper the method of imaging of wideband audio sources based on the 2D microphone array measurements of the sound field at the same time in all the microphones is proposed. Designed microphone array consists of 160 microphones allowing to digitize signals with a frequency of $7200 \mathrm{~Hz}$. Measured signals are processed using the special algorithm that makes it possible to obtain a flat image of wideband sound sources. It is shown experimentally that the visualization is not dependent on the waveform, but determined by the bandwidth. Developed system allows to visualize sources with a resolution of up to $10 \mathrm{~cm}$.
\end{abstract}

\section{Introduction}

Detection and visualization of sound sources is of great practical importance $[1,2]$ for remote vibration diagnostics, sound quality studies and creation of remote microphones. In addition, it is possible to monitor and determine a coordinates of speaking people [3], which is important for security. The task of arbitrary audio sources imaging is solved on the basis of simultaneous measurement of the sound field at different points in space. It is desirable to place the sensors with spatial gaps less than half of a wavelength. The aperture should be of the order of distance to the test sound source. There are solutions [3], to track speaking people and to distinguish between them by voice. For the calibration of ultrasonic transducers used multiposition scanners. The solution of the inverse problem of wave propagation considering evanescent waves allows to determine the near-zone emitter field [4]. However, in this case, time synchronization is required between the transmitter and receiver. In case of a third-party audio sources detection, a priori about them is no information, and it is impossible to synchronize the transmitter and receiver, and thus provide estimation of the phase at the point of measurement. To preserve the spatial coherence of the measured field necessary to measure simultaneously the signals at various points. Unfortunately, it is impossible to synchronize to arbitrary time source, and if there may be several sources, and each emits its signal. When measured on a flat aperture to rely only on the presence of in-plane resolution, while to obtain range resolution fails. Nevertheless, even the flat images are of great practical importance.

\footnotetext{
${ }^{\text {a }}$ Corresponding author: sdy@mail.tsu.ru
} 
In this paper, we propose to carry out the focus of the measured field in the object plane at each frequency separately and then sum up the intensities of the received monochromatic images. This approach allows us to visualize any sound sources regardless of the waveform.

\section{Visualization of sound sources}

The scheme of the broadband measurements on the basis of a two-dimensional array of microphones shown in Figure 1. A two-dimensional array of microphones located in front of the sound source at a distance $h$. We assume that a third-party source emits a signal with a wide frequency range. Radiated waves fall into the microphone array and simultaneously digitized. It is assumed that the range to the emitter is less than or about the aperture size of the system. Thus, the source is in the near area of array, and therefore applying of wave field focusing it is possible sources visualization with a wavelength resolution.

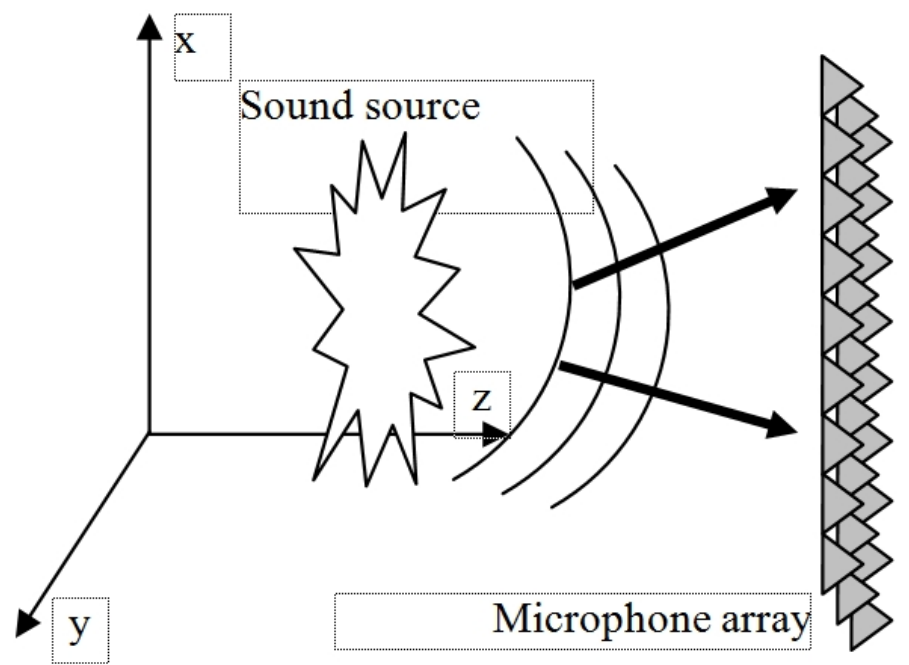

Figure 1. Scheme of ultrasonic measurements.

To solve the inverse problem of accurate sources images recovery we propose a method of broadband spatial matched filtering. First apply the Fourier transform to the signals measured from all microphones:

$$
\widetilde{P}(x, y, z=0, \omega)=\int_{-\infty}^{\infty} P(x, y, z=0, t) \exp (i \omega t) d t,
$$

where $P(x, y, z=0, t)$ - signal, measured in point $(x, y, 0), \omega-$ frequrncy.

Since the measurements are simultaneous, the spatial coherence of signals stored on separate frequencies. Next, we calculate the plane wave spectrum of the wave field at each frequency in the measurement plane:

$$
\widetilde{\widetilde{P}}\left(k_{x}, k_{y}, z=0, \omega\right)=\int_{-\infty}^{\infty} \int_{-\infty}^{\infty} \widetilde{P}(x, y, z=0, \omega) e^{-i k_{x} x-i k_{y} y} d x d y,
$$

where $k_{x}, k_{y}$ - spatial frequencies. 
By the plane-wave spectrum, we can easily restore the field in the source plane [57]. We are restoring the spatial spectrum of the source in the plane at a distance $h$ by a factor $\exp \left(-i k_{z} h\right)$ :

$$
\widetilde{P}(x, y, z=-h, \omega)=\int_{-\infty}^{\infty} \int_{-\infty}^{\infty} \widetilde{\widetilde{P}}\left(k_{x}, k_{y}, z=0\right) \exp \left(-i k_{z} h\right) \exp \left(i k_{x} x+i k_{y} y\right) d k_{x} d k_{y},
$$

where $k_{z}=\sqrt{(\omega / c)^{2}-k_{x}{ }^{2}-k_{y}{ }^{2}}$. Modifier $\exp \left(-i k_{z} h\right)$ allows us to restore even field and evanescent waves in the near-source zone. Because there is no synchronization of the sound source and the receiver, we can not provide in-phase addition of the signals from the source at all frequencies. It is therefore proposed to sum up the squares of modules of ultrasound images at each frequency. Square module, but not module itself, proposed to use as it will increase the level of the dominant signal relative to the background, which helps visualize concentrated sources. Thus, wideband processing is performed as follows:

$$
P\left(x_{s}, y_{s}\right)=\sum_{n}\left|\widetilde{P}\left(x_{s}, y_{s}, \omega_{n}\right)\right|^{2}
$$

The proposed method has been verified by numerical simulations. The numerical model considered two point sources at a distance of $60 \mathrm{~cm}$ from the measurement plane. Figure 2 shows the simulated measured field at a frequency of $3500 \mathrm{~Hz}$. It is considered that radiation produced in the frequency band from $500 \mathrm{~Hz}$ to 3500 . A shape of the signal is random. Two emitters are not synchronized and emit different signals.

Note, however, that the field is not measured at all points in space, but only where the microphones are. Consider the case of the 96 array elements arranged in a rectangular grid $16 \times 6$ cells with gaps of $6 \mathrm{~cm}$. Location of the microphones is shown in Figure 3.

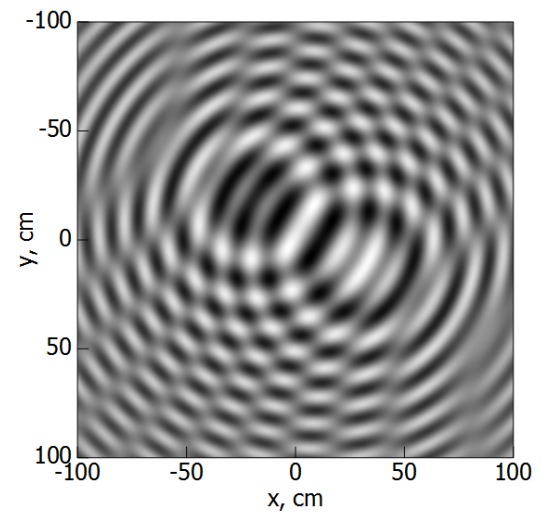

Figure 2. Field of sources on $3500 \mathrm{~Hz}$.

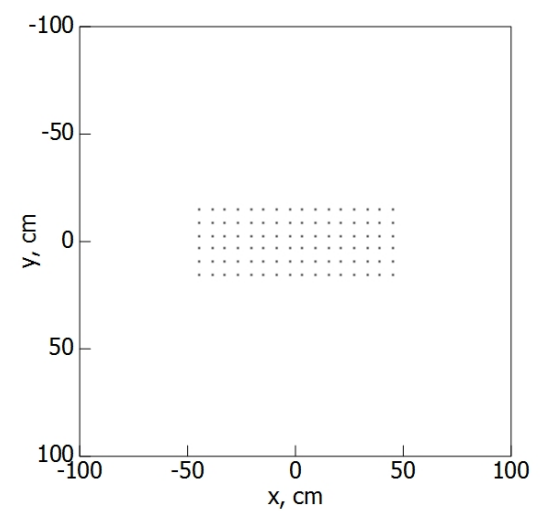

Figure 3. Placement of microphones in receiver array.

After processing by the broadband space - matched filter the intensity image of two sources has been restored (Figure 4). Image has artifacts caused by array sparsity. Image sources localized stronger along the $x$ axis than along the $y$ axis because of the aperture size of $x$ axis is $90 \mathrm{~cm}$ and on the axis $y$ is $30 \mathrm{~cm}$. After treatment by the formula (4) the image of two broadband sources was restored (Figure 5). 


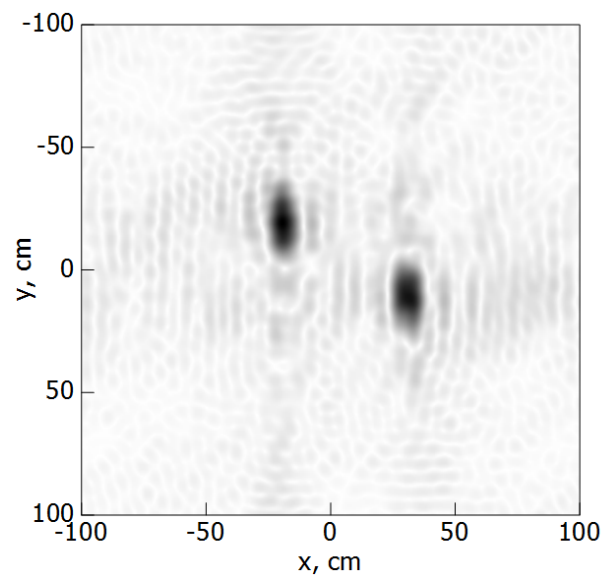

Figure 4. Reconstruction of sources image at a distance $60 \mathrm{~cm}$ on frequency $3500 \mathrm{~Hz}$.

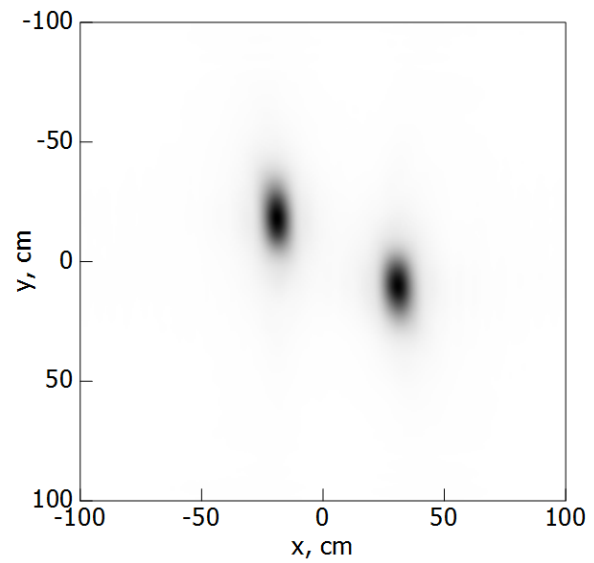

Figure 5. Reconstruction of sources image at a distance $60 \mathrm{~cm}$ in an frequency range $500-3500$ $\mathrm{Hz}$.

In this image there are no artifacts due to the fact that the sum intensity increases the signal - noise at $\sqrt{N}$ times. numerical simulation results confirm the applicability of the method of broadband space - matched filtering.

\section{Experimental research}

For experimental studies two-dimensional array of microphones has been developed consisting of 6 linear arrays (Figure 6).
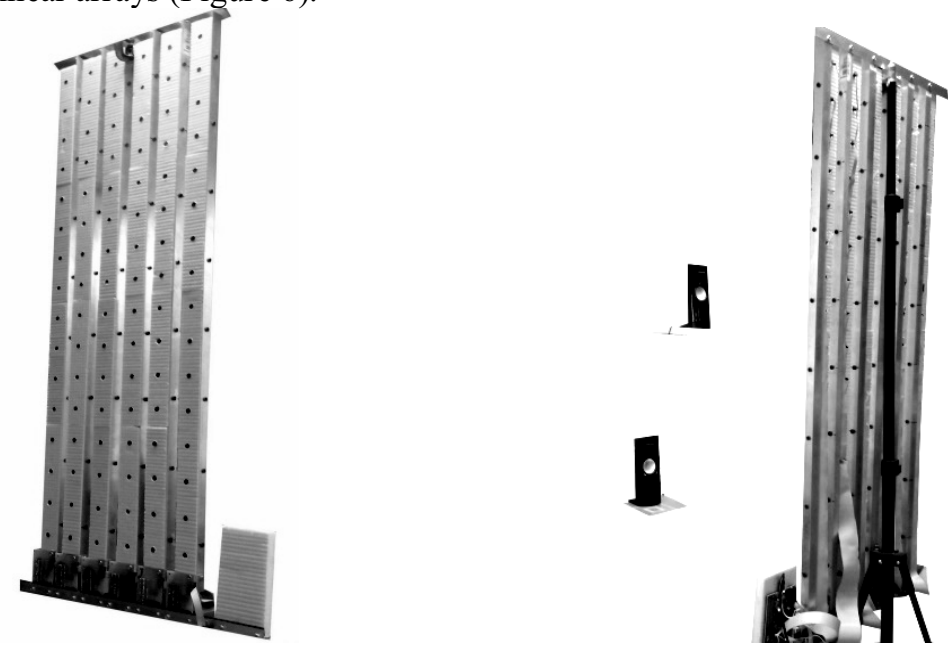

Figure 6. Photo of experimental setup.

Each line contains a set of 16 electret microphones connected to a separate unit with a 16-channel multiplexer and an amplifier. Linear arrays are connected via a multiplexer to the ADC block individual channels. Each electret microphone bias voltage is supplied via a separate resistor. Out of a separate microphone connected via a capacitor to the multiplexer. The multiplexer is connected to the microcontroller STM32F407 and controlled by four binary outputs addressing and one output on / off. Multiplexer output is connected to a microcontroller, which allows the digitized signal from all the microphones in the linear 
array. This setting allows you to simultaneously digitize signals of a 96 microphones with a frequency of $7200 \mathrm{~Hz}$. Microcontroller transmits digitized signals to a personal computer for further processing. A two-dimensional array of the microphone is positioned vertically to the sound source at a distance of $60 \mathrm{~cm}$. The sound source is a speaker that emits a signal at a particular frequency range and controlled by the computer. As a result of the experiment was measured noise signal (frequency band from 100 to $4000 \mathrm{~Hz}$ ) being emitted from the speakers and fall into one of the microphones is shown in Figure 7.

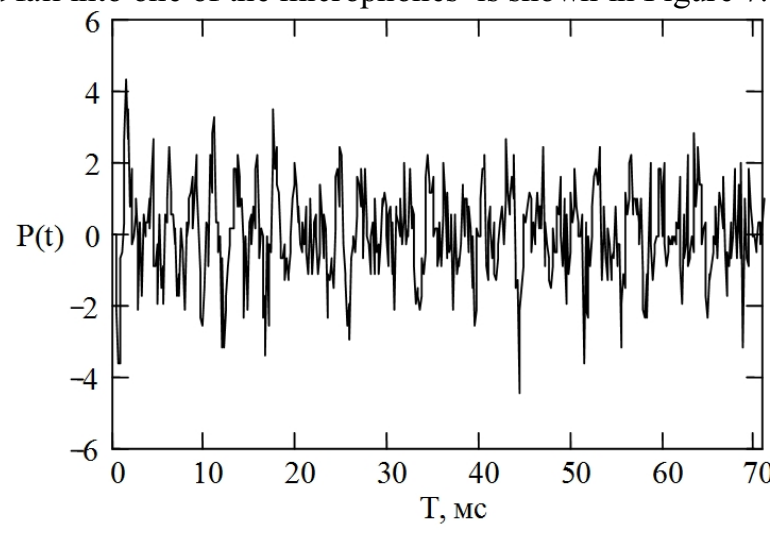

Figure 7. Noise signal in a microphone array from the two oscillators.

It is known that the spectrum of the noise signal is approximately uniform at all frequencies, which means that the signal is broadband. Formula (1-4), whereby the image was restored two sound sources (see Figure 8 ) have been applied to the measured data.

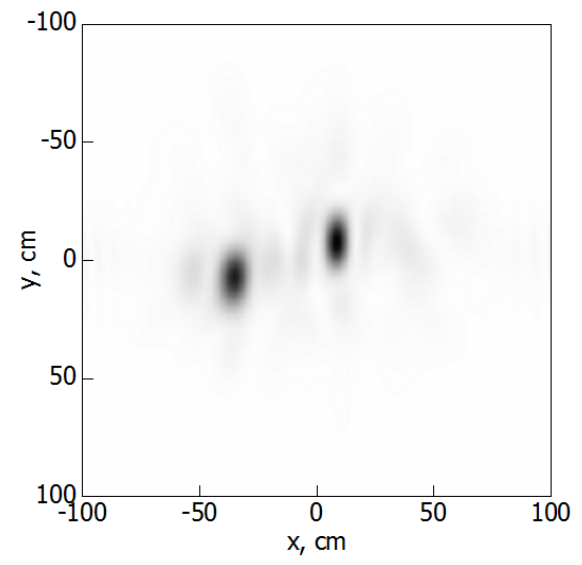

Figure 8. Wideband noise signal Image from the sound sources.

It can be seen that the experimental result is in good agreement with numerical simulations. Point emitters clearly visualized. That is, there is a possibility of detecting the quite arbitrary signal source. It does not require a priori information about the signal form but only enough signal to be broadband. The experimental setup provides a resolution of about $10 \mathrm{~cm}$. 


\section{Conclusion}

The proposed method of recovery of homogeneous and heterogeneous fields near a sound source zone unsynchronized with the receiver, allows you to create a tool for studying the dynamics of the field of near-field ultra-wideband sources. To apply the method requires simultaneous measurements of audio signals of two-dimensional array of microphones. This method has been verified by numerical simulations and experiments. The experimental setup consists of 96 microphones, allowing to digitize signals at a frequency of $7200 \mathrm{~Hz}$. The experimental study confirmed the applicability of the developed method, the experimental data are consistent with the results of the numerical model.

\section{Acknowledgment}

The research is supported by Russian Science Foundation project No 16-19-10272.

\section{References}

[1] P. Gregush, Ultrasonic Imaging: Seeing by Sound (Focal Press, London, 1980)

[2] V. P. Yakubov, S. E. Shipilov; D. Ya. Sukhanov, Russian Physics Journal 55, 878 (2013) doi: 10.1007/s11182-013-9895-9

[3] A. Quinlan, M. Kawamoto, Y. Matsusaka, et. al., Eurasip Journal on Audio, Speech, and Music Processing 2009, 673202 (2009) doi : 10.1155/2009/673202

[4] E.G. Williams, J.D. Maynard, Phys. Rev. Lett. 45, 554 (1980) doi: 10.1103/PhysRevLett.45.554

[5] P.R. Stepanishen, K.S. Benjamin, Journal of the Acoustical Society of America 71, 803 (1982) doi : 10.1121/1.387606

[6] Yong Thung Cho M.J. Roan, J.S. Bolton, Proc. IMechE Part C: J. Mechanical Engineering Science 223,819 (2009) doi: 10.1243/09544062JMES1209

[7] D.Ya. Sukhanov, N.N. Erzakova, CriMiCo 2014, 950 (2014) doi: 10.1109/CRMICO.2014.6959709 